\title{
25 Research Soure \\ A Distance-Based Microfluidic Paper-Based Biosensor for Glucose Measurements in Tear Range
}

\section{Samira Allameh}

University of Isfahan Faculty of Engineering

Mohsen Rabbani ( $\sim$ m.rabbani@eng.ui.ac.ir)

University of Isfahan Faculty of Engineering https://orcid.org/0000-0002-9187-076X

\section{Research Article}

Keywords: Microfluidic paper-based analytical device ( $\mu \mathrm{PAD})$, Glucose, Colorimetric detection, CO2 laser, paper, Tears

Posted Date: November 16th, 2021

DOl: https://doi.org/10.21203/rs.3.rs-522698/v2

License: (c) (i) This work is licensed under a Creative Commons Attribution 4.0 International License. Read Full License

Version of Record: A version of this preprint was published at Applied Biochemistry and Biotechnology on January 14th, 2022. See the published version at https://doi.org/10.1007/s12010-022-03817-8. 


\section{Abstract}

The prevalence of diabetes has increased over the past years. Therefore, developing minimally invasive, userfriendly, and cost-effective glucose biosensors is necessary, especially in low-income and developing countries. Cellulose paper-based analytical devices have attracted the attention of many researchers due to affordability, not requiring trained personnel, and complex equipment. This paper describes a microfluidic paper-based analytical device for detecting glucose in tear range with the naked eye. The paper-based biosensor fabricated by laser $\mathrm{CO}_{2}$ and GOx/HRP enzymatic solution coupled with TMB was utilized as reagents. A sample volume of $10 \mu \mathrm{l}$ was needed for the biosensor operation, and the results were observable within 5 minutes. The color intensity-based and distance-based results were analyzed by ImageJ and Tracker to evaluate the device performance. Distancebased results showed a linear behavior in 0.1-1.2 $\mathrm{mM}$ with an $\mathrm{R}^{2}=0.9962$ and LOD of $0.1 \mathrm{mM}$. The results could be perceived by the naked eye without needing additional equipment or trained personnel in a relatively short time (35 minutes). Moreover, glucose concentration could be obtained non-invasively by tears collected by this $\mu$ PAD.

\section{Introduction}

Diabetes is a metabolic disorder that is the major reason for mortality and health-related problems in developing countries ${ }^{1,2}$. According to the World Health Organization (WHO), about 422 million people have diabetes all over the world ${ }^{3}$. The self-monitoring of blood glucose, which can be obtained by glucose biosensors, is one way to control this disease ${ }^{4}$. Other biofluids such as saliva, tear, sweat, interstitial fluid (ISF), and urine can be used to obtain the blood glucose concentration ${ }^{5}$. The main reason for utilizing these biofluids is to avoid finger prick in the elderly, newborns, and hemophiliacs ${ }^{6}$. The important point about alternative biofluids is the correlation of the glucose analyte in the biofluid and blood ${ }^{7}$.

In comparison with other biofluids, the tear is more accessible and does not require further preparation. Whereas low glucose concentration in tear and low volume of tear, are some of the disadvantages of using the tear as biofluid in glucose sensors ${ }^{8-11}$. The glucose concentration in the tear in various studies is different due to the method of sampling ${ }^{12}$. According to a study, a tear-based glucose biosensor should have a linear behavior in 0.1 $3 \mathrm{mM}^{8}$.

Microfluidics has attracted the attention of many biosensor researchers over the last decade. Sensors that have microfluidic technology can detect molecules in small sample volumes. Reducing the sample volume, response time, and improving sensitivity are some of the positive features of microfluidic diagnostic devices ${ }^{13,14}$. WHO established ASSURED guidelines for such devices, which stand for Affordable, Sensitive, Specific, User-friendly, Rapid and Robust, Equipment-free, and Deliverable to the end-users ${ }^{15}$.

According to such guidelines, paper is an appropriate substrate for microfluidic devices due to the low cost, availability even in low-resources countries, the capability to perform chemical reactions on the substrate and achieving acceptable sensitivity, not requiring preparation when performing assays, and fluid wicking through capillary action without the need to external pumps ${ }^{15-18}$. Microfluidic paper-based analytical devices ( $\mu$ PADs) were firstly introduced by Whitesides in $2007^{19}$. HPADs are widely used in colorimetric assays to detect glucose, uric acid, and other analytes in medicine. Easy immobilization of reagents is another advantage of the porous structure of paper, e.g., immobilization of glucose oxidase through a simple adsorption mechanism ${ }^{17,20,21}$. 
Colorimetric assay is the most common detection method in the applications of $\mu$ PADs because of several advantages, such as employing affordable equipment for image acquisition (e.g., smartphone cameras, standard scanners, digital cameras, or portable microscopes). Color inhomogeneity is a challenge in colorimetric assays ${ }^{12}$.

For the glucose colorimetric detection, glucose oxidation occurs in the presence of glucose oxidase (GOx) enzyme, and hence hydrogen peroxide $\left(\mathrm{H}_{2} \mathrm{O}_{2}\right)$ and gluconic acid are produced $16,22,23$. The second reaction is based on the

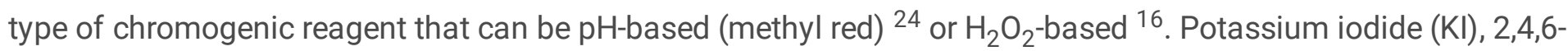
tribromo-3-hydroxybenzoic acid (TBHBA), 4-aminoantipyrine (4-AAP), 3,5-dichloro-2-hydroxy-benzenesulfonate (DHBS), 3-aminopropyltriethoxysilane (APTMS), 3,3'-diaminobenzidine (DAB), and 3,3',5,5'-

tetramethylbenzidine (TMB) are some of the famous chromogenic reagent for $\mathrm{H}_{2} \mathrm{O}_{2}{ }^{16}$. When $\mathrm{H}_{2} \mathrm{O}_{2}$-based reagent is used, $\mathrm{HRP}$ catalyzes the reaction of chromogenic reagent, and the $\mathrm{H}_{2} \mathrm{O}_{2}$ and a colored product are produced $16,22,23$.

In the colorimetric assay, the results can be analyzed by naked-eye or color analysis softwares such as ImageJ 25 , GIMP ${ }^{24}$, Quantity one software ${ }^{26}$, and Adobe Photoshop ${ }^{19} 27$. RGB ${ }^{24}$ and grayscale ${ }^{28}$ images are mostly utilized in various studies. In the naked-eye colorimetric methods, the results are analyzed without needing complex equipment or software ${ }^{5}$. Time-based methods, ladder bar-based detection, and distance-based methods are some of the naked-eye detection assays in which the concentration of the analyte is related to the analysis time, the number of colored columns, and the length of the color change, respectively ${ }^{29-31}$. In the distance-based methods, the analyte quantification is carried out by the length of the color change. To decrease the individual error and dependence of results to the user ${ }^{31}$. It can also adapt the dynamic range by changing the reagent concentration and geometry 29 .

For fabricating a cellulose paper-based device, cost and resolution are the most important factors for selecting the method. There are various methods for the fabrication of $\mu$ PADs. These fabrication techniques are classified into two general classifications: physical and chemical ${ }^{32}$. In physical fabrication techniques, a hydrophobic material is added to the paper, and hydrophilic channels are developed, whereas, in chemical methods, the hydrophilic property of the paper is changed by adding chemical materials ${ }^{32,33}$.

In laser cutting, the cellulose paper is cut, and a two-dimensional device is fabricated. The high resolution, not requiring hydrophobic materials for channel fabrication, rapid fabrication, and mass production are the interesting features of laser $\mathrm{CO}_{2}$ cutting. A disadvantage of this physical fabrication technique is the need for expensive equipment and careful selection of the power and laser rate to prevent paper burning ${ }^{32,33}$. The paper-based device which is fabricated by the laser, is not as rigid as $\mu$ PADs fabricated by other methods. Thus, a plastic film as backing or sealing is required for the packaging. Packaging has various advantages, such as preventing $\mu P A D$ contamination, sample evaporation, and leakage. Pressure-sensitive tapes are primarily utilized as sealing films 33.

This study aims to design and fabricate a colorimetric glucose $\mu$ PAD for tear analysis. The device is fabricated from cellulose filter paper by $\mathrm{CO}_{2}$ laser, and pressure-sensitive adhesive tape is utilized for sealing. GOx, HRP, and TMB are reagents, and tear range concentrations of glucose solutions are used. The assay is recorded by a smartphone camera while the color intensity-based and distance-based results are analyzed by ImageJ (in 
greyscale) and Tracker, respectively. Various factors such as the type of chromogenic reagent, immobilization, method of adding TMB and its concentration, and other factors are investigated.

\section{Materials And Methods}

\subsection{Materials and chemical agents}

Glucose oxidase (GOx) (Merck, Germany) from aspergillus niger, horseradish peroxidase (HRP) (Merck, Germany), 3,3,5,5-tetramethylbenzidine (TMB) (BioBasic Inc, Canada), glucose (Merck, Germany), Polyvinylpyrrolidone (PVP) (Merck, Germany), Potassium lodide (KI), Silica nanoparticle (SiNP, Fadak, Iran), methanol (Arman Sina, Iran), and Phosphate Buffered Saline (PBS, pH=7.6) (Merck, Germany) were purchased. All chemicals were used as received. Filter paper $\left(80 \mathrm{~g} / \mathrm{m}^{2}\right)$ with $50 \%$ porosity and transparent pressure-sensitive adhesive tape (TPSA) were used as the substrate (hydrophilic channel) and sealing film, respectively.

\subsection{Equipment and software}

$\mathrm{A} \mathrm{CO}_{2}$ laser engraving cutting machine (Perfect, China) operating at a wavelength of $10.64 \mu \mathrm{m}$ at $14 \mathrm{~W}$ was utilized for cutting cellulose filter paper. Images and videos were captured by a smartphone camera (iPhone 11, USA). CorelDRAW (2018), Tracker (5.1.3), and ImageJ (1.52a) were used for the channel design, distance-based measurement, and intensity-based image processing, respectively. The images were captured in a specific room and under a particular light and finally subtracted by a defined value in ImageJ to eliminate the background effect.

\section{3 $\mu$ PAD design and fabrication}

Hydrophilic patterns were designed in CorelDRAW 2019. The device consisted of a sample zone $\left(2 \times 5 \mathrm{~mm}^{2}\right)$, primary circular zone (diameter $=5 \mathrm{~mm}$ ), detection zone (channel, $2 \times 25 \mathrm{~mm}^{2}$ ), and absorbent circular zone (diameter $=5 \mathrm{~mm}$ ). The designed $\mu \mathrm{PAD}$ and different zones are demonstrated in Fig. 1a. The patterns were cut by a $\mathrm{CO}_{2}$ laser engraving cutting machine $(\lambda=10.64 \mu \mathrm{m}, 14 \mathrm{~W}, 14 \mathrm{~mm} / \mathrm{s})$. Reagents were added to different zones, and the device was packed with a regular TPSA to prevent leakage and evaporation the $\mu$ PAD.

\subsection{Enzymatic reactions of glucose colorimetric detection}

The device was dipped in a PVP solution to improve the immobilization of the chemical agents. An enzymatic solution containing $120 \mathrm{U} / \mathrm{ml}$ of GOx and $30 \mathrm{U} / \mathrm{ml}$ of HRP was added to the primary circular zone. The chromogenic solution containing TMB was dissolved in methanol and added to the channel called the detection zone. The role of the final circular zone was absorbing the extra fluid. The zone of the enzymatic solution and chromogenic agents was investigated in various experiments, and adding them to the primary circular zone and channel, respectively, was selected to achieve acceptable distance-based results.

Different concentrations of $10 \mu \mathrm{l}$ glucose solution were added to the sample zone and wicked towards the primary circular zone by capillary action. Glucose was oxidized in the presence of GOx and the products (gluconic acid and hydrogen peroxide) entered the detection zone. $\mathrm{H}_{2} \mathrm{O}_{2}$ reacted with TMB in the presence of HRP, and the blue 
oxidized TMB was produced in the detection zone. The second circular zone absorbed excessive water and byproducts. The schematic of the reactions is illustrated in Fig. 1b, Fig. 1c and Fig. 1d.

\subsection{Procedure for color detection}

The colorimetric assay video was captured by a smartphone camera and analyzed by ImageJ and Tracker to achieve intensity-based and distance-based results, respectively. For the intensity-based results, the frame of the video was imported into ImageJ and digitized to grayscale.

The cellulose paper is white, and the color intensity decreases after the chemical reaction, so more glucose concentration results in lower color intensity. The inverse grayscale was used to directly correlate color intensity and glucose concentration (inverse grayscale $=255$ - grayscale).

The video was imported in Tracker for the distance-based results. A vector determined the wetted length or colored length at different time intervals ( 0 to $240 \mathrm{~s}, 0 \mathrm{~s}$ assumed the time that sample entered the channel). The initial and terminal points of the vector were the beginning of the channel and the end of the wetted or colored area (Fig. 3b). Finally, the vector magnitude over time diagram was plotted.

The wetted-length diagram showed the possibility of the glucose sample wicking feasibility along the channel, whereas the colored-length diagram indicated the presence of glucose and its reaction with other reagents. Therefore, when the wetted length is more than the colored length, it means the glucose had been finished during the reactions, and the sample without glucose was wicked towards the end of the channel, as can be observed in Fig. 1e.

\subsection{Evaluation of different parameters}

Various parameters were investigated to realize the performance of the $\mu P A D$ through color intensity-based and distance-based results. The Chromogenic reagent, the immobilizer, method of adding the TMB reagent, TMB concentration, and the enzymatic solution volume were investigated.

\subsubsection{Type of chromogenic reagent}

TMB (15 mM) and KI (1.2 M) were used as chromogenic reagents. The color intensity and distance-based results were the evaluation factors. Each test was carried out four times for statistical clarity.

\subsubsection{Type of the immobilization}

The disadvantage of not using an immobilizer is that the color change could flow along the channel, and consequently, the intensity-based and distance-based results could be inaccurate. Therefore, three types of immobilizations were utilized, and wetted length, colored length, and color intensity were compared with the immobilization-free experiment.

The immobilization agents were PVP solution (concentration 1\%, dissolved in PBS) and SiNP (10-15 and $100 \mathrm{~nm}$, dispersed in deionized water). Each test was carried out four times for statistical clarity.

\subsubsection{Method of adding TMB}


The amount of chromogenic solution can affect the colored-length and intensity-based results. Four methods of adding TMB were defined:

1. Adding solution by a micropipette uniformly as much as the channel became wet; it is called the uniform method (UM).

2. Adding the solution by a micropipette every $5 \mathrm{~mm}$ of the channel; it is called the non-uniform method (NUM).

3. Adding solution twice by a micropipette uniformly with an interval of 5 minutes to allow the first series of TMB solution to be dried; it is called twice uniform method (2UM).

4. Dipping the secondary circular zone in the TMB solution and allowing the TMB to wick into the channel; it is called the saturated method (SM) because most of the paper pores were filled and saturated with the chromogenic solution.

Each test was carried out four times for statistical clarity.

\subsubsection{The concentration of TMB}

Different concentrations of TMB solutions $(2.5,3,3.75,5 \mathrm{mM})$ were used as chromogenic reagents to have an appropriate colored length for the tear glucose concentration. Each test was carried out four times for statistical clarity.

\subsubsection{The volume of enzymatic solution}

The enzymatic solutions were added to the primary circular zone to investigate the effect of the enzyme volume on the color intensity-based results. For this purpose, adding $3 \mu \mathrm{l}, 4 \mu \mathrm{l}$, and dipping the circular zone in enzymatic solution (saturated method, SM) were examined to obtain adequate volume for the solution. Each test was carried out four times for statistical clarity.

\subsubsection{Distance-based biosensor evaluation}

According to previous experiments, the final test was carried out by adding the $3 \mu$ of the enzymatic solution to the primary circular zone, and the channel of the device was saturated with TMB solution, as the chromogenic factor, with the concentration of $3.75 \mathrm{mM}$. Various concentrations of glucose samples in tear range $(0.1,0.2,0.4$, 0.8 , and $1.2 \mathrm{mM}$ ) were added to the sample zone, and the colored length was measured. Each test was carried out four times for statistical clarity.

\section{Results And Discussion}

\subsection{Evaluation of different factors}

Various factors were evaluated while color intensity-based and distance-based results were obtained considering the test type to achieve an appropriate performance for the $\mu \mathrm{PAD}$ glucose biosensor.

\subsubsection{Type of chromogenic reagent}

$\mathrm{TMB}$ and $\mathrm{KI}$ were used as the chromogenic solution, and glucose solutions with a concentration of $2.5,5,10$, and $20 \mathrm{mM}$ were used as samples (only length-based results of $10 \mathrm{mM}$ glucose sample are displayed to reduce the article volume). The velocity of the glucose solution (slope of the plot) in the wetted-length plot (Fig. 2a), was more 
when TMB was used as a chromogenic solution and it reached the end of the channel earlier. Moreover, the color change occurred immediately after adding the glucose sample in the presence of TMB, while for $\mathrm{KI}$ it occurred 3 minutes after the sample addition; therefore, the colored bar for KI was showed up at $120 \mathrm{~s}$ (Fig. 2b). As indicated in the color intensity diagram (Fig. 2c), the color intensity was more when KI was used as a chromogenic solution, but the color intensity discrepancy was low. As explained in length and intensity-based results, the TMB solution is used for further analysis.

\subsubsection{Type of the immobilizer}

Three types of immobilizers (PVP solution and SiNP in different sizes of 10-15 nm and $100 \mathrm{~nm}$ ) were utilized for $2.5,5,10$, and $20 \mathrm{mM}$ of glucose (only results of $10 \mathrm{mM}$ are depicted). As indicated in Fig. $\mathbf{3 a}$, the immobilizer affected the sample velocity and color intensity. The glucose solution could reach the end of the channel faster in an immobilizer-free assay. Moreover, increasing nanoparticle size resulted in the deceleration of glucose solution wicking along the channel when SiNP was utilized as an immobilizer. The color change occurred faster when the immobilization agent was not used (Fig. 3b). According to the color intensity diagram (Fig. 3c), using a PVP solution for immobilization enhanced the color intensity compared to other assays. Besides, when the immobilizer was not utilized, the color change could flow along the channel and affect the distance-based results. Therefore, the PVP solution is used for future investigations

\subsubsection{Method of adding TMB}

Four methods of adding TMB by the concentration of $15 \mathrm{mM}$ (UM, NUM, 2UM, and SM) were investigated for 2.5, 5,10 , and $20 \mathrm{mM}$ of glucose (only results of $10 \mathrm{mM}$ are depicted). As illustrated in Fig. $4 \mathrm{a}$, the velocity of the sample decreased with increasing TMB owing to the reduction of pores that help fluid wicking. Additionally, this decrease could be observed for the colored-length diagrams in the assays with more TMB due to the consumption of the whole glucose in a shorter channel length. In UM assay, the final colored length equaled the channel length, which shows that all the glucose did not participate in the reaction, and consequently, the amount of TMB was not adequate for such amount of glucose (Fig. 4b).

When a colored-length diagram for various glucose concentrations in the SM is plotted, a gentle behavior can be observed between the colored length and glucose concentration. According to Fig. 4c, the colored length did not change after the 150s. The colored length in a specified time (150 s) over the glucose concentration is illustrated in Fig. 4d. Since the tear glucose is less than $1.2 \mathrm{mM}$ and also the colored length for this concentration is under 5 $\mathrm{mm}$, the concentration of TMB solution should be calibrated. The effect of TMB concentration is investigated in future tests. The color intensity for different assays was approximately the same except when TMB was added nonuniformly (NUM) because of the lower TMB solution volume and finally the lower products and color change (Fig. 4e).

\subsubsection{The concentration of TMB}

TMB solutions with concentrations of $2.5,3,3.75$, and $5 \mathrm{mM}$ were evaluated. The glucose concentration was in the tear glucose range (0.1-1.2 mM). As mentioned in previous experiments (Method of adding TMB), results could

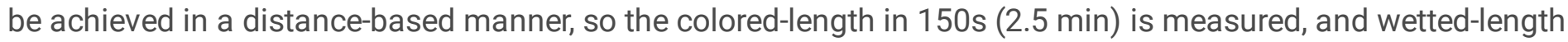
and colored-length results are neglected. As demonstrated in Fig. 5a, the colored length increased by reducing the TMB concentration. This is actually due to the earlier running out of the glucose in more TMB concentration through the reaction and consequently very low colored length; therefore, the concentration of $5 \mathrm{mM}$ TMB was not 
appropriate for the tear range glucose. The colored length for $2.5,3$, and $3.75 \mathrm{mM}$ were very similar. The color intensity was increased by increasing the TMB concentration (Fig. 5b). The concentration of $3.75 \mathrm{mM}$ of TMB solution is selected for further tests because of more colored length and higher color intensity.

\subsubsection{The volume of the enzymatic solution}

The enzymatic solution volumes of $3 \mu \mathrm{l}, 4 \mu \mathrm{l}$, and SM were examined. According to the colored-length over glucose concentration diagram, using a lower amount of enzymatic solution offered more colored length (Fig. 6a). The color intensity was decreased by increasing the enzymatic solution amount (Fig. $\mathbf{6 b}$ ). Therefore, $3 \mu$ l of the enzymatic solution was selected.

\subsubsection{Distance-based glucose detection assay}

The glucose solution with a concentration of 0.1-1.2 mM was utilized. The colored length showed a linear relationship to glucose concentration. The linear equation obtained colored length $=13.4 \times$ (glucose concentration) -0.7653 with an $\mathrm{R}^{2}=0.9962$.

\section{Conclusion}

Diabetes is a common disorder all over the world. Self-monitoring of blood glucose is one of the ways of controlling diabetes and is carried out by glucose biosensors ${ }^{2,14}$. Nowadays, $\mu$ PADS are widely used in medicine, the environment, and food monitoring because of various features such as paper substrate material abundancy, fluid flow through capillary action passively, easy enzyme immobilization, and etc $17,24,34$. A minimally invasive glucose biosensor was fabricated by a $\mathrm{CO}_{2}$ laser in the present study to determine the tear range glucose sample through a distance-based colorimetric assay. The enzymatic solution contained GOx $120 \mathrm{U} / \mathrm{ml}$ and HRP $30 \mathrm{U} / \mathrm{ml}$. TMB with a concentration of $3.75 \mathrm{mM}$ was selected as a chromogenic solution. The reagents were added to the specified zones after dipping the $\mu \mathrm{PAD}$ in an immobilizer (1\% PVP). The wetted-length, colored-length, and inverted grayscale color intensity were measured by using this $\mu \mathrm{PAD}$ assay. Various concentrations of $10 \mu \mathrm{l}$ glucose solution were added to the sample zone. For the distance-based assay, the colored length was measured at a specific time (150s). Since the color change of the TMB solution is unstable, the naked-eye results could be observed within $5 \mathrm{~min}$. The dynamic range of the device was $0.1-1.2 \mathrm{mM}$ with an LOD of $0.1 \mathrm{mM}$. The summary of recent studies and comparison with this study is demonstrated in Table 1.

\section{Declarations}

The authors declare that they have no known competing financial interests or personal relationships that could have appeared to influence the study reported in this paper.

\section{Funding}

The authors disclosed receipt of the following financial support for the research, authorship, and/or publication of this article: This work was supported by the University of Isfahan. 


\section{Conflict of interests}

Authors have no conflict of interests to declare.

\section{Authors' contributions}

Samira Allameh was MSc. Student in biomedical engineering and she did this study as a master thesis under the supervision of Dr. Mohsen Rabbani. Samira wrote the manuscript and Mohsen modified and prepared it for publication.

\section{Availability of data and material}

All useful data obtained during the study are available.

\section{Ethics approval (Not applicable)}

This research does not contain any studies with human participants or animals performed by any of the authors.

\section{Consent to participate (Not applicable)}

This research does not contain any studies with human participants performed by any of the authors.

\section{Consent for publication(Not applicable)}

\section{Acknowledgments}

The team acknowledges the generous funding provided by the University of Isfahan, Isfahan, Iran.

\section{References}

1. Turner, A. \& Fragkou, V. Commercial biosensors for diabetes. in Taylor \& Francis Group 41-64 (2008). doi:10.1201/9781584889755.ch2.

2. Martinkova, P. \& Pohanka, M. Biosensors for blood glucose and diabetes diagnosis: evolution, construction, and current status. Anal. Lett.48, 2509-2532 (2015).

3. World Health Organization. Health topics, diabetes. (2020).

4. Yoo, E. H. \& Lee, S. Y. Glucose biosensors: An overview of use in clinical practice. Sensors10, 4558-4576 (2010).

5. Radhakumary, C. \& Sreenivasan, K. Naked eye detection of glucose in urine using glucose oxidase immobilized gold nanoparticles. Anal. Chem.83, 2829-2833 (2011).

6. Jung, D. G., Jung, D. \& Kong, S. H. A lab-on-a-chip-based non-invasive optical sensor for measuring glucose in saliva. Sensors (Switzerland)17, 1-12 (2017). 
7. Dominguez, R. B., Orozco, M. A., Chávez, G. \& Márquez-Lucero, A. The evaluation of a low-cost colorimeter for glucose detection in salivary samples. Sensors (Switzerland)17, 19-21 (2017).

8. Lane, J. D., Krumholz, D. M., Sack, R. A. \& Morris, C. Tear glucose dynamics in diabetes mellitus. Curr. Eye Res.31, 895-901 (2006).

9. Zhang, J., Hodge, W., Hutnick, C. \& Wang, X. Noninvasive diagnostic devices for diabetes through measuring tear glucose. J. Diabetes Sci. Technol.5, 166-172 (2011).

10. Bandodkar, A. J. \& Wang, J. Non-invasive wearable electrochemical sensors: A review. Trends Biotechnol.32, 363-371 (2014).

11. La Belle, J. T. et al. Self-monitoring of tear glucose: The development of a tear based glucose sensor as an alternative to self-monitoring of blood glucose. Chem. Commun.52, 9197-9204 (2016).

12. Gabriel, E. F. M., Garcia, P. T., Lopes, F. M. \& Coltro, W. K. T. Paper-based colorimetric biosensor for tear glucose measurements. Micromachines8, (2017).

13. Demirci, U., Khademhosseini, A., Langer, R. \& Blander, J. Microfluidic technologies for human health. World Scientific (2012). doi:10.1142/8469.

14. Selmi, M., Gazzah, M. H. \& Belmabrouk, H. Optimization of microfluidic biosensor efficiency by means of fluid flow engineering. Sci. Rep.7, 1-11 (2017).

15. Walji, N. Characterization of fluid flow in paper-based microfluidic devices. (Master thesis, University of Ontario, Canada, 2015).

16. Liu, S., Su, W. \& Ding, X. A review on microfluidic paper-based analytical devices for glucose detection. Sensors (Basel).16, (2016).

17. Chandra Sekar, N., Mousavi Shaegh, S. A., Ng, S. H., Ge, L. \& Tan, S. N. A paper-based amperometric glucose biosensor developed with Prussian Blue-modified screen-printed electrodes. Sensors Actuators, B Chem.204, 414-420 (2014).

18. Songok, J. \& Toivakka, M. Enhancing capillary-driven flow for paper-based microfluidic channels. ACS Appl. Mater. Interfaces8, 30523-30530 (2016).

19. Chen, X. et al. Determination of glucose and uric acid with bienzyme colorimetry on microfluidic paper-based analysis devices. Biosens. Bioelectron.35, 363-368 (2012).

20. Zhou, M., Yang, M. \& Zhou, F. Paper based colorimetric biosensing platform utilizing cross-linked siloxane as probe. Biosens. Bioelectron.55, 39-43 (2014).

21. Kuek Lawrence, C. S., Tan, S. N. \& Floresca, C. Z. A 'green' cellulose paper based glucose amperometric biosensor. Sensors Actuators, B Chem.193, 536-541 (2014).

22. Gabriel, E. F. M. et al. Highly sensitive colorimetric detection of glucose and uric acid in biological fluids using chitosan-modified paper microfluidic devices. Analyst141, 4749-4756 (2016).

23. Morbioli, G. G., Mazzu-Nascimento, T., Stockton, A. M. \& Carrilho, E. Technical aspects and challenges of colorimetric detection with microfluidic paper-based analytical devices ( $\mu$ PADs) - A review. Anal. Chim. Acta970, 1-22 (2017).

24. Soni, A. \& Jha, S. K. A paper strip based non-invasive glucose biosensor for salivary analysis. Biosens. Bioelectron.67, 763-768 (2015).

25. Fatoni, A., Anggraeni, M. D. \& Dwiasi, D. W. Simple colorimetric glucose biosensor using chitosan cryogel supporting material. AIP Conf. Proc.1746, (2016). 
26. Zhu, W. J. et al. Bienzyme colorimetric detection of glucose with self-calibration based on tree-shaped paper strip. Sensors Actuators, B Chem.190, 414-418 (2014).

27. Nery, E. W. \& Kubota, L. T. Sensing approaches on paper-based devices: A review. Anal. Bioanal. Chem.405, $7573-7595$ (2013).

28. Jin, L. et al. Ultrasmall Pt nanoclusters as robust peroxidase mimics for colorimetric detection of glucose in human serum. ACS Appl. Mater. Interfaces9, 10027-10033 (2017).

29. Tian, T. et al. Distance-based microfluidic quantitative detection methods for point-of-care testing. Lab Chip16, 1139-1151 (2016).

30. Lewis, G. G., Ditucci, M. J. \& Phillips, S. T. Quantifying analytes in paper-based microfluidic devices without using external electronic readers. Angew. Chemie - Int. Ed.51, 12707-12710 (2012).

31. Cate, D. M., Dungchai, W., Cunningham, J. C., Volckens, J. \& Henry, C. S. Simple, distance-based measurement for paper analytical devices. Lab Chip13, 2397-2404 (2013).

32. He, Y., Wu, Y., Fu, J. Z. \& Wu, W. Bin. Fabrication of paper-based microfluidic analysis devices: a review. RSC Adv.5, 78109-78127 (2015).

33. Yetisen, A. K., Akram, M. S. \& Lowe, C. R. Paper-based microfluidic point-of-care diagnostic devices. Lab Chip13, 2210-2251 (2013).

34. Fan, Y., Liu, S., Gao, K. \& Zhang, Y. Fully enclosed paper-based microfluidic devices using bio-compatible adhesive seals. Microsyst. Technol.24, 1783-1787 (2018).

35. Kang, B. H., Park, M. \& Jeong, K. H. Colorimetric Schirmer strip for tear glucose detection. Biochip J.11, 294299 (2017).

36. Santana-Jiménez, L. A., Márquez-Lucero, A., Osuna, V., Estrada-Moreno, I. \& Dominguez, R. B. Naked-eye detection of glucose in saliva with bienzymatic paper-based sensor. Sensors (Switzerland)18, 1-12 (2018).

\section{Tables}

Table 1 The summary of recent studies and comparison with this study 


\begin{tabular}{|c|c|c|c|c|c|}
\hline Novelty & $\begin{array}{l}\text { - Glucose } \\
\text { measurement } \\
\text { in tear range }\end{array}$ & $\begin{array}{l}\text { - Using } \\
\text { Schirmer } \\
\text { test } \\
\text { strips as } \\
\text { the } \\
\text { substrate }\end{array}$ & & $\begin{array}{l}\text { - Glucose } \\
\text { measurement } \\
\text { without need } \\
\text { to equipment }\end{array}$ & $\begin{array}{l}\text { - Low } \\
\text { concentrations } \\
\text { of glucose } \\
\text { measurement } \\
\text { without need } \\
\text { to equipment } \\
\text { - Possibility of } \\
\text { sampling by } \\
\text { the device } \\
\text { - Easy } \\
\text { fabrication } \\
\text { technique } \\
\text { without } \\
\text { requirements } \\
\text { to } \\
\text { hydrophobes } \\
\text { - }\end{array}$ \\
\hline $\begin{array}{l}\text { Fabrication } \\
\text { method }\end{array}$ & Wax printing & Wax printing & $\begin{array}{l}\text { Stamp } \\
\text { patterning }\end{array}$ & Wax printing & $\mathrm{CO}_{2}$ cutting laser \\
\hline $\begin{array}{l}\text { Analyze } \\
\text { method }\end{array}$ & $\begin{array}{l}\text { Color intensity } \\
\text { (Photo-Paint } \\
\text { software) }\end{array}$ & $\begin{array}{l}\text { Color } \\
\text { intensity }\end{array}$ & $\begin{array}{l}\text { Color } \\
\text { intensity } \\
\text { (GIMP } \\
\text { software) }\end{array}$ & Distance based & Distance based \\
\hline $\begin{array}{l}\text { Assay } \\
\text { duration } \\
\text { (min) }\end{array}$ & 15 & - & - & $<10$ & 2.5 \\
\hline LOD (mM) & 0.5 & 0.1 & 0.05 & 0.6 & 0.1 \\
\hline $\begin{array}{l}\text { Dynamic } \\
\text { range } \\
\text { (mM) }\end{array}$ & $0.1-1$ & $0.1-1.4$ & $0.05-1.5$ & $0.6-15$ & $0.1-1.2$ \\
\hline Reagent & $\begin{array}{l}\text { GOx, HRP, TMB, } \\
\text { Chitosan }\end{array}$ & $\begin{array}{l}\text { GOx, HRP, 4- } \\
\text { AAp }\end{array}$ & $\begin{array}{l}\text { GOX, } \\
\text { HRP,TBHBA, } \\
\text { 4-AAP }\end{array}$ & $\begin{array}{l}\text { GOx, HRP, DAB, } \\
\text { AgNP }\end{array}$ & $\begin{array}{l}\text { GOx, HRP, TMB, } \\
\text { PVP }\end{array}$ \\
\hline $\begin{array}{l}\text { Sample } \\
\text { volume }(\mu l)\end{array}$ & 5 & 6 & 2 & 20 & 10 \\
\hline \multirow[t]{2}{*}{ Sample } & Tear & Tear & Saliva & Human serum & $\begin{array}{l}\text { Glucose solution in } \\
\text { tear range }\end{array}$ \\
\hline & 22 & 35 & 36 & 31 & Present study \\
\hline
\end{tabular}

\section{Figures}


Primary circular zone

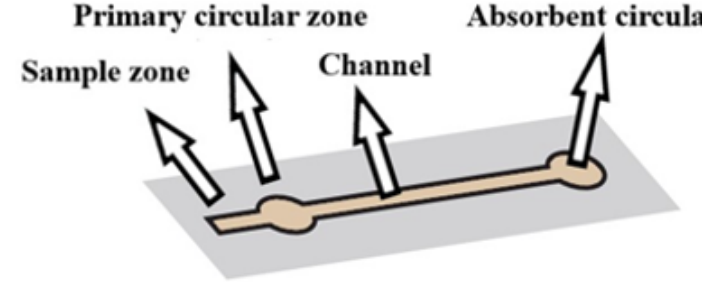

(a)

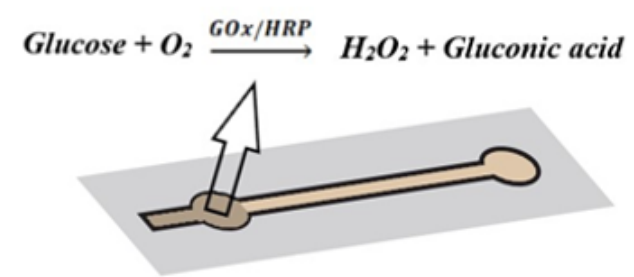

(c)

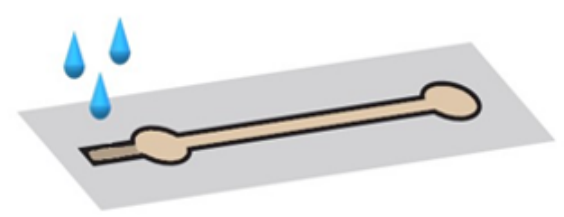

(b)

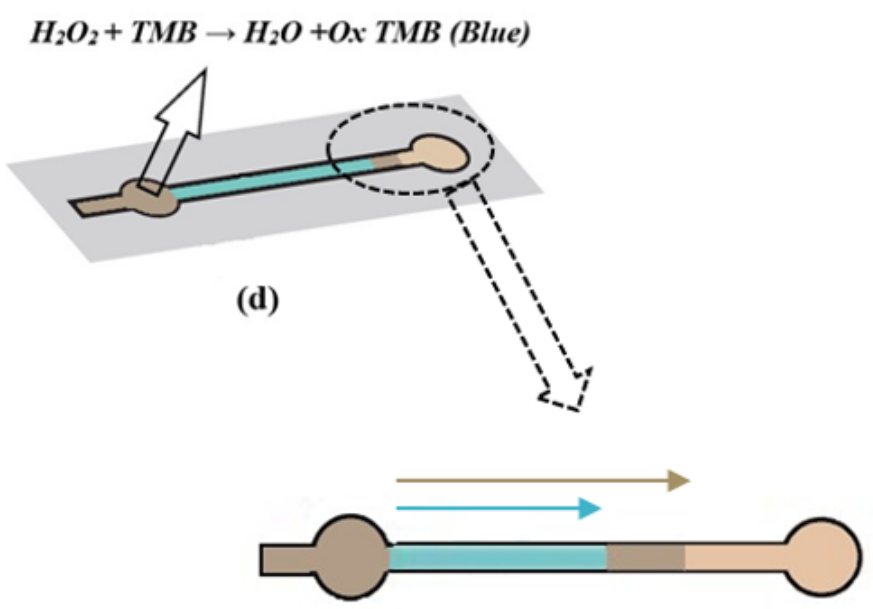

(e)

\section{Figure 1}

The simplified schematic of the colorimetric reaction on the designed device in the presence of enzymatic solution (GOx and HRP) and chromogenic reagent (TMB) a) Schematic view of the designed device and different zones $b$ ) Addition of glucose sample to the device c) Glucose oxidation in the prescence of enzymes and $\mathrm{H} 2 \mathrm{O} 2$ production d) $\mathrm{H} 2 \mathrm{O} 2$ reaction with TMB and production of blue oxidized TMB e) The colored length and the wetted length vectors are displayed in blue and brown vectors, respectively 
(a)

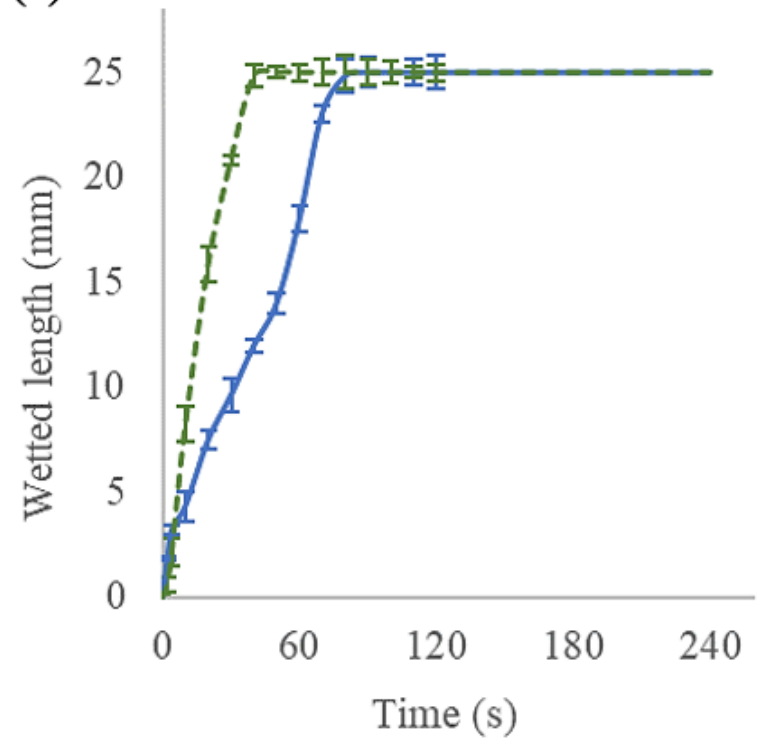

(b)

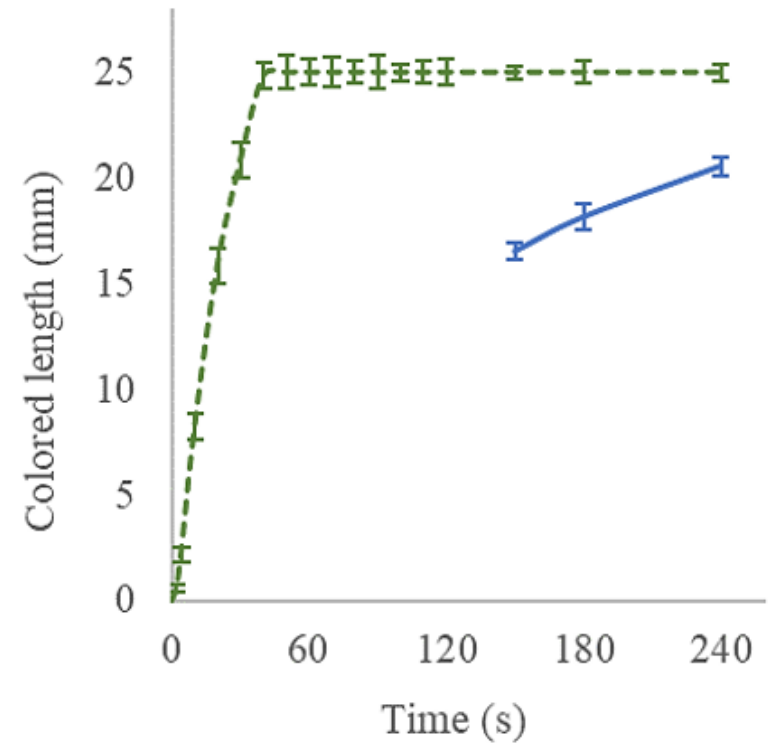

- KI solution

TMB solution

(c)

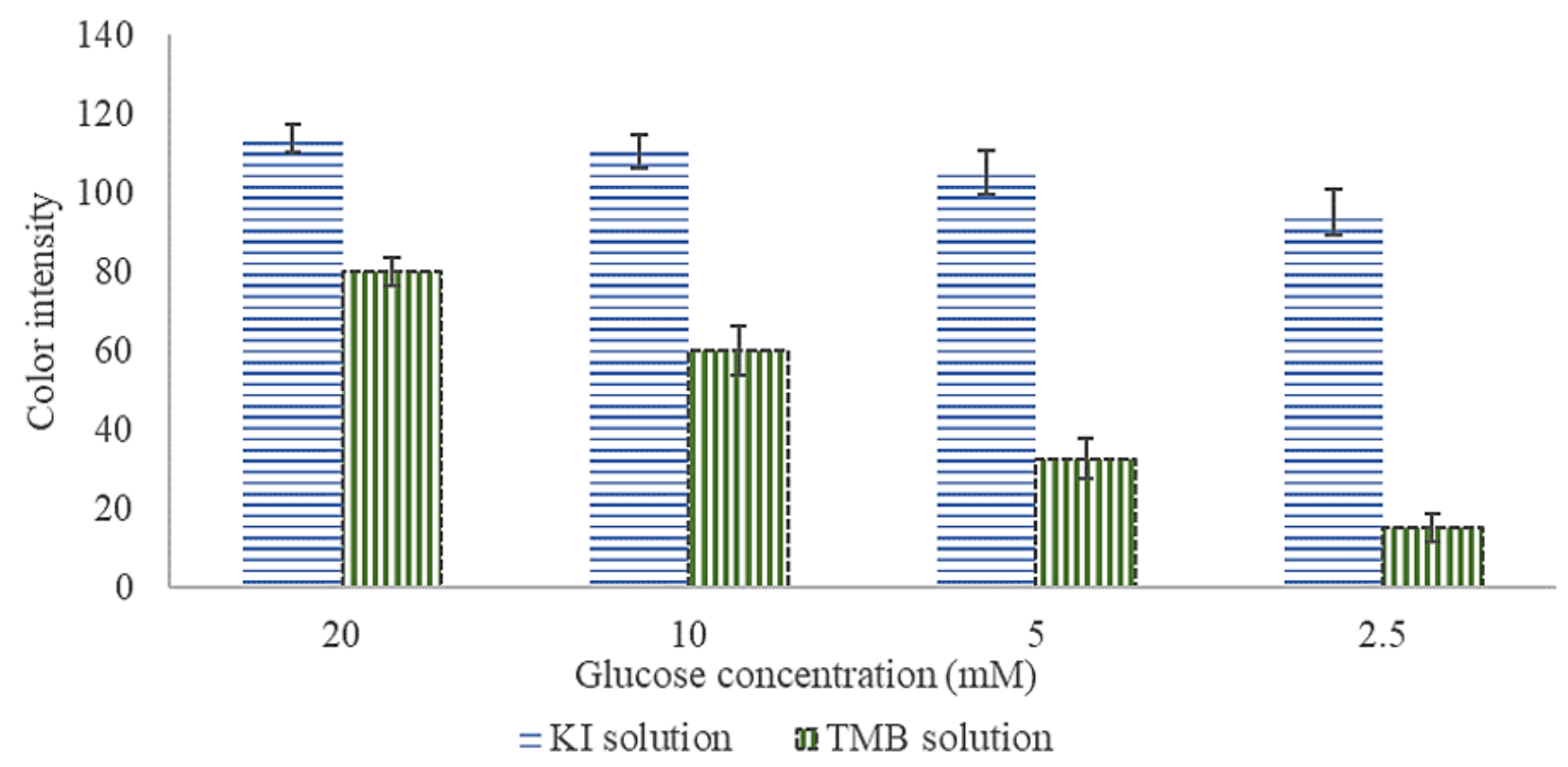

Figure 2

a) Wetted-length diagram for $10 \mathrm{mM}$ of glucose solution, the solution moved faster in the presence of TMB compared with $\mathrm{KI}$ solution b) Colored-length diagram for $10 \mathrm{mM}$ of glucose solution, earlier color change in TMB solution compared with $\mathrm{KI}$ solution c) Color intensity diagram for various glucose concentrations, more discrepancy in color intensity of various concentrations when TMB solution is used as a chromogenic reagent 
(a)

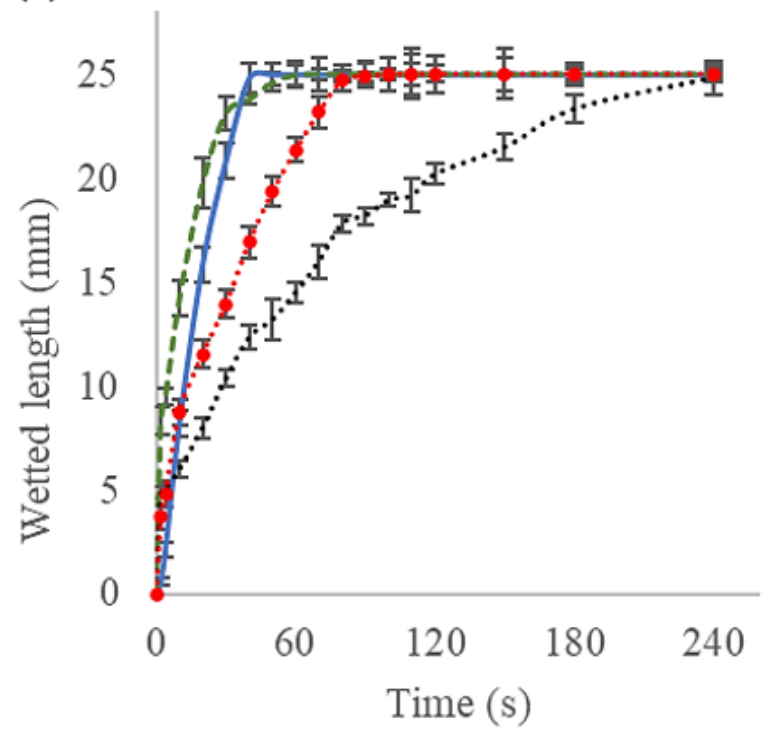

(b)

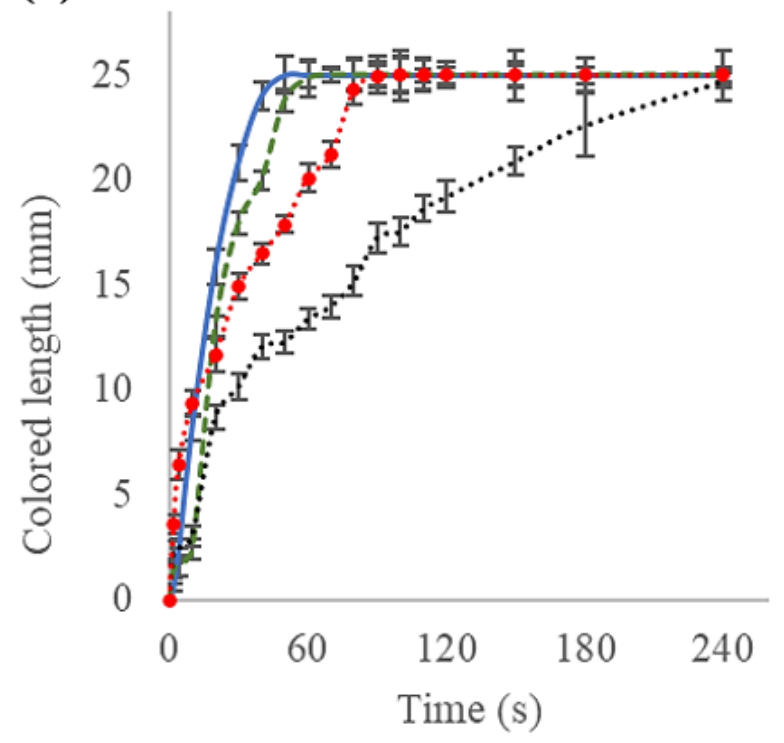

— Immobilization-free $\quad-----\operatorname{SiNP}(10-15 \mathrm{~nm}) \quad \cdots \cdots \cdots \cdot \operatorname{SiNP}(100 \mathrm{~nm}) \quad \cdots \cdot \cdots \cdot$ PVP

(c)

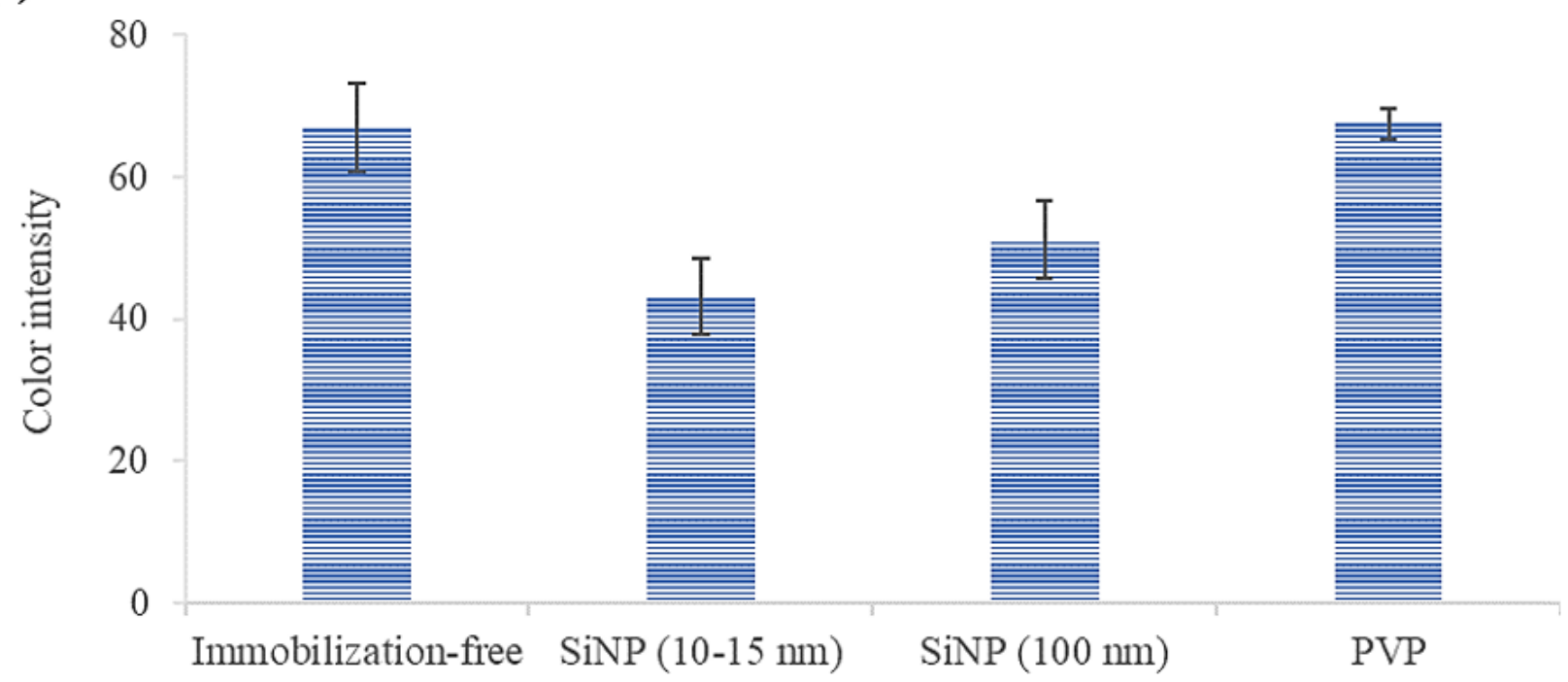

Figure 3

a) Wetted-length diagram for $10 \mathrm{mM}$ of glucose when the effect of immobilization was examined, faster wicking and reaching the end of the device channel when immobilization was not used b) Colored-length diagram, faster color change in immobilizer-free assay c) The greyscale color intensity when various immobilization solutions were used for $10 \mathrm{mM}$ of glucose, the higher grayscale intensity in the detection zone when PVP solution was used as an immobilizer 
(a)

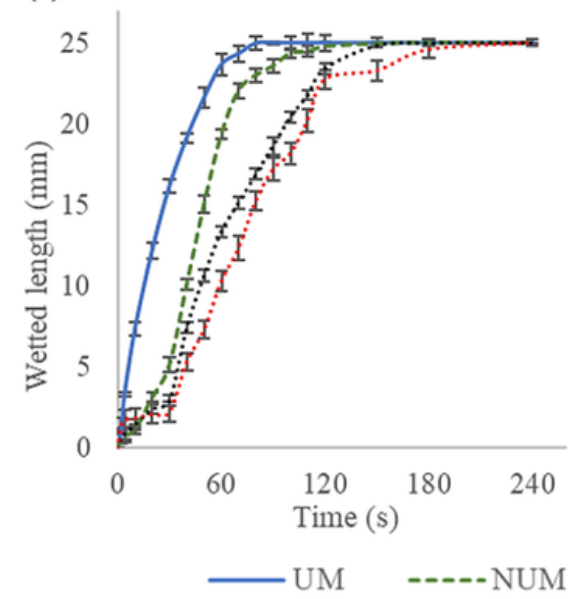

(c)

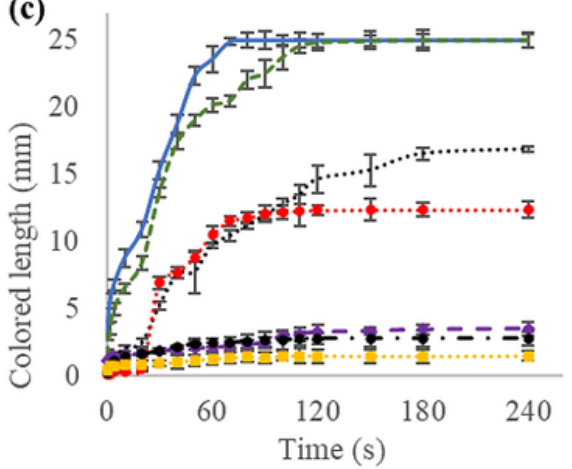

$20 \mathrm{mM} \quad-----10 \mathrm{mM} \quad \cdots \cdots+\ldots . .5 \mathrm{mM}$

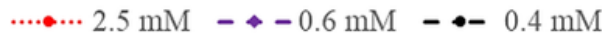

$\cdots \cdots 0.2 \mathrm{mM}$

(e)

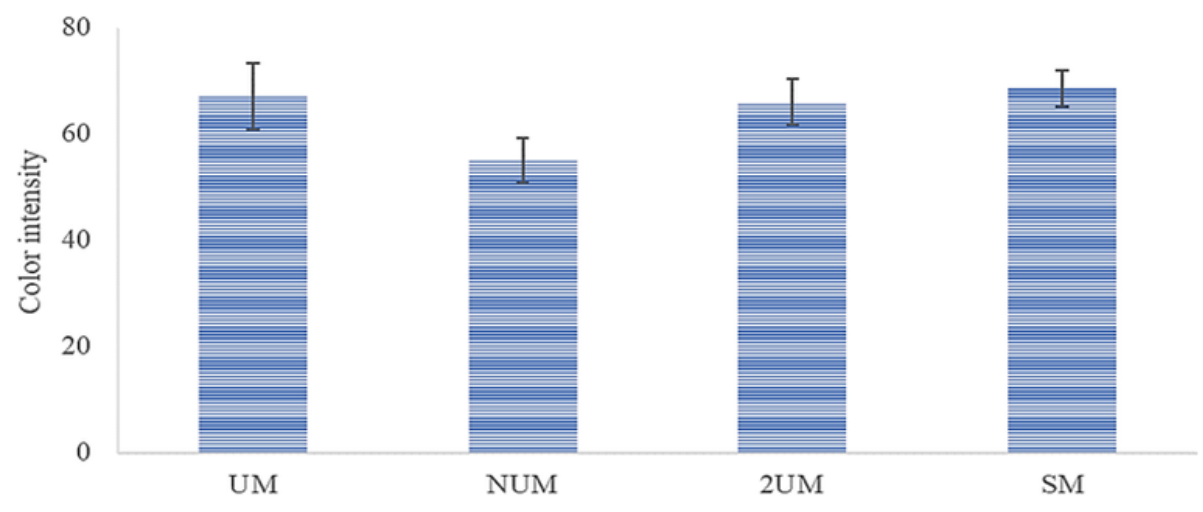

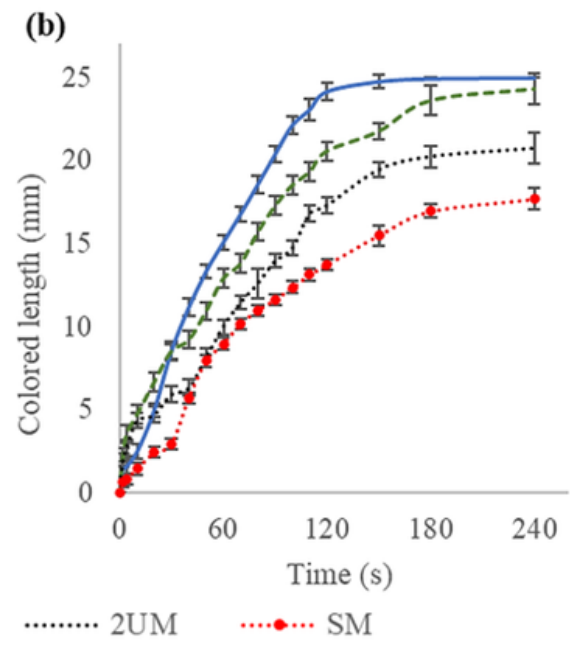

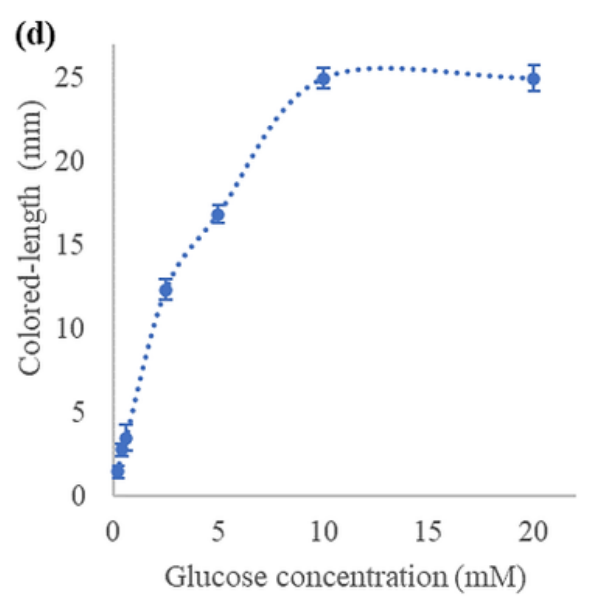

\section{Figure 4}

a) Wetted-length diagram in the method of adding TMB solution experiments for $10 \mathrm{mM}$ of glucose, faster wicking of glucose sample when lower TMB solution was used b) Colored-length diagram, decrease colored length in the presence of more TMB as earlier running out of the glucose $\mathrm{c}$ ) Colored-length over time diagram for various glucose concentrations, colored length increased in the presence of more glucose concentration d) Colored length diagram for various glucose concentrations in 150s, more colored length was observed in the presence of more glucose concentration e) Color intensity diagram for different methods of adding TMB solution for $10 \mathrm{mM}$ of glucose, lower color intensity correlated with lower TMB solution volume 
(a)

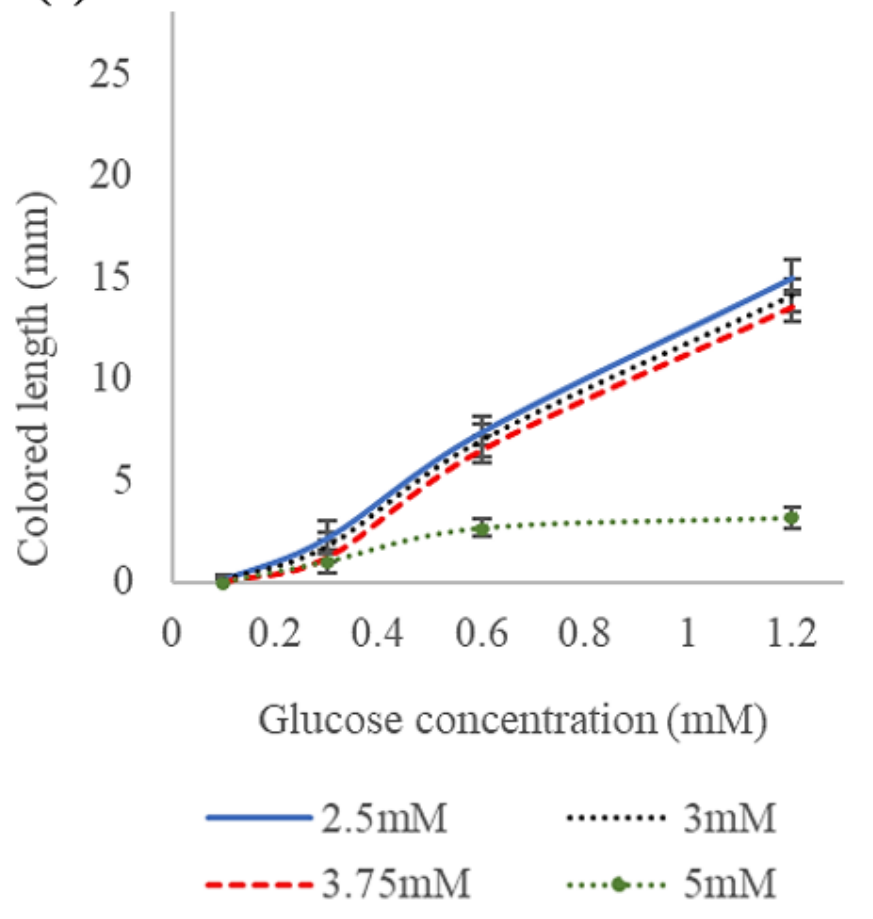

(b)

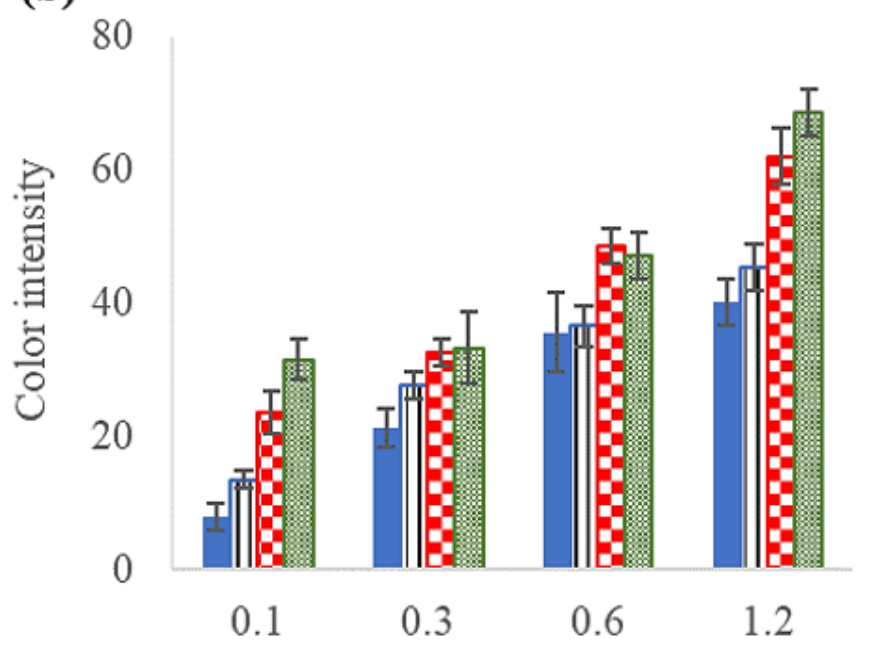

Glucose concentration (mM)
$\square 2.5 \mathrm{mM}$
由 $3 \mathrm{mM}$
口 $3.75 \mathrm{mM}$

\section{Figure 5}

a) Colored-length diagram in 150s when different TMB concentrations were used, more colored length in lower TMB concentration b) Color intensity diagram, more grayscale intensity for the $3.75 \mathrm{mM}$ of TMB

(a)

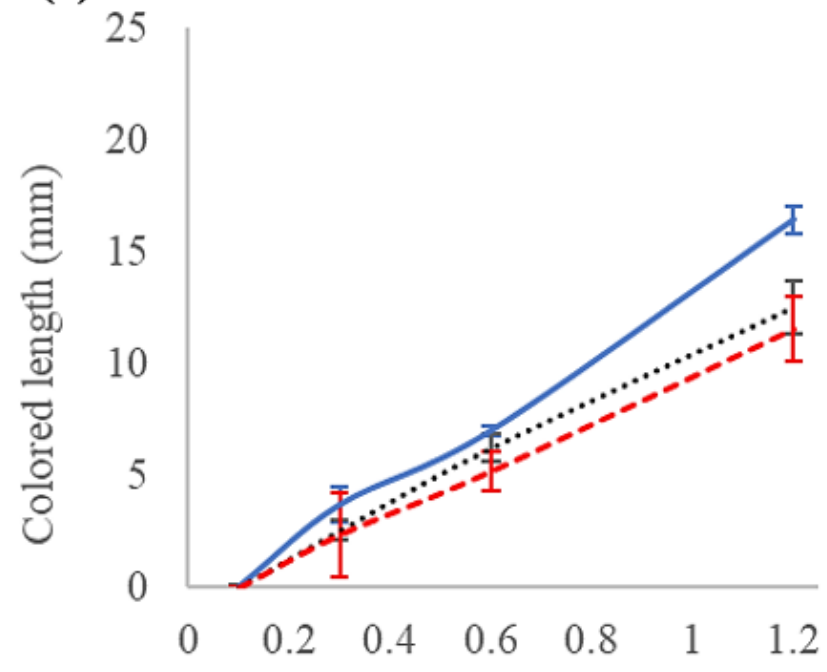

Glucose concentration (mM)

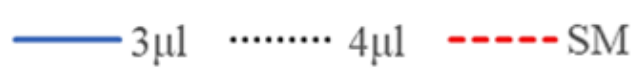

(b)

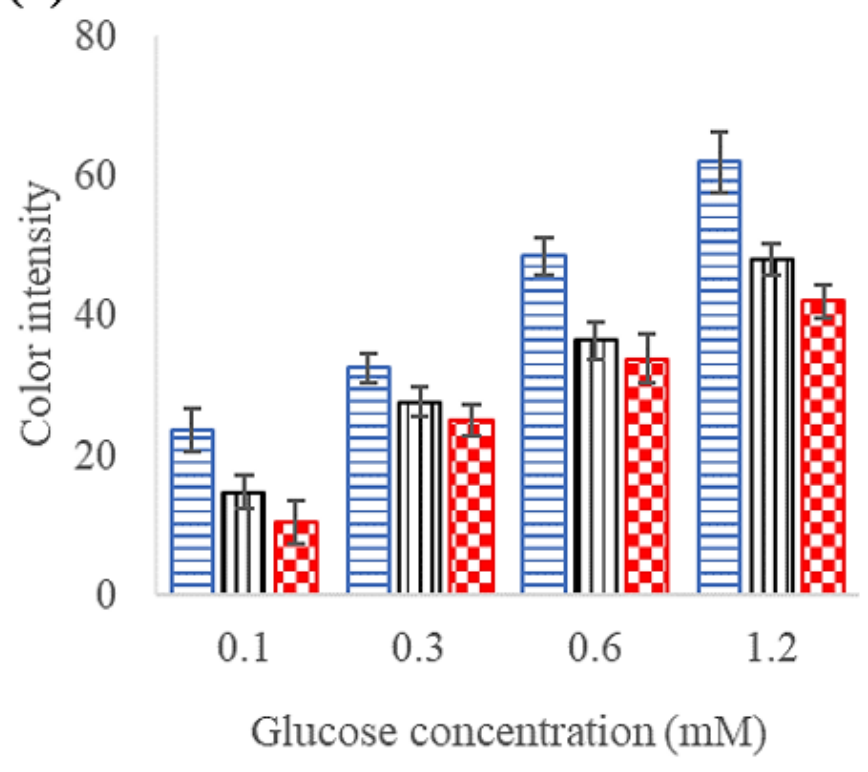

분 $\square 4 \mu \mathrm{l} \quad \mathrm{SM}$

Figure 6 
a) Colored-length diagram in 150 s when different volumes of enzymatic solution were used, increasing colored length by decreasing enzymatic solution volume b) More color intensity by lowering amount of enzymatic solution in various glucose concentrations

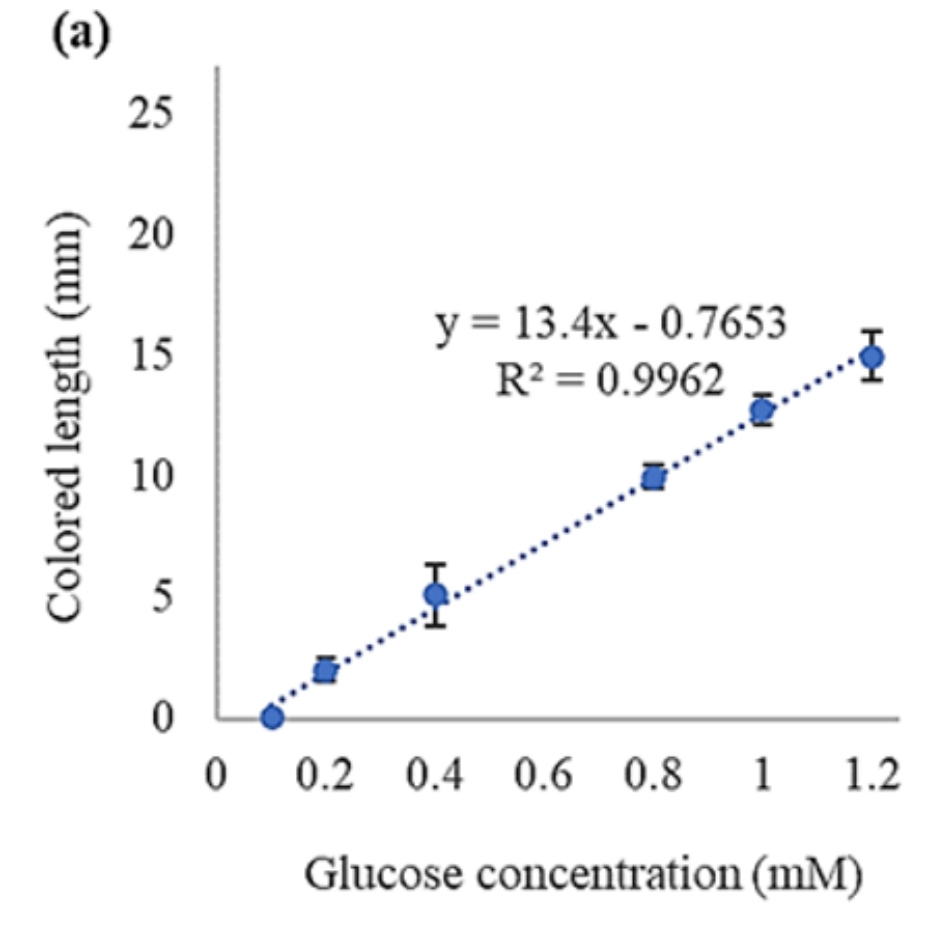

(b)

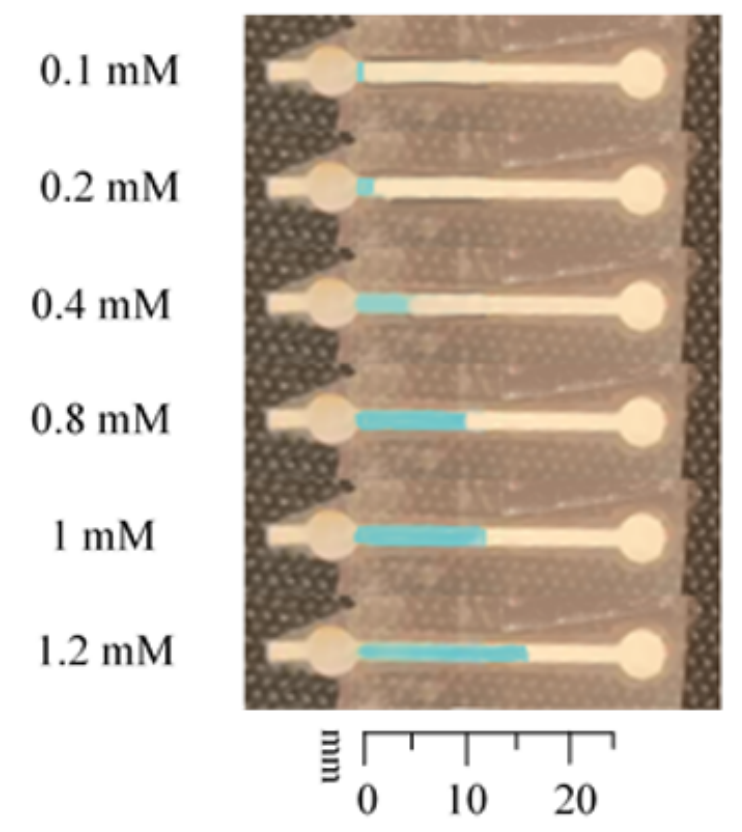

Figure 7

a) The linear behavior of the device for different glucose concentrations in tear range b) Different colored length in various glucose concentrations 